\title{
Some common random fixed point theorems for contractive type conditions in cone random metric spaces
}

\author{
Gurucharan S. Saluja \\ Department of Mathematics, \\ Govt. Nagarjuna P. G. \\ College of Science, India \\ email: saluja1963@gmail.com
}

\author{
Bhanu Pratap Tripathi \\ Department of Mathematics, \\ Govt. Nagarjuna P. G. \\ College of Science, India \\ email: bhanu.tripathi@gmail.com
}

\begin{abstract}
In this paper, we establish some common random fixed point theorems for contractive type conditions in the setting of cone random metric spaces. Our results unify, extend and generalize many known results from the current existing literature.
\end{abstract}

\section{Introduction}

Random nonlinear analysis is an important mathematical discipline which is mainly concerned with the study of random nonlinear operators and their properties and is needed for the study of various classes of random equations. The study of random fixed point theory was initiated by the Prague school of Probabilities in the 1950s [9, 10, 24]. Common random fixed point theorems are stochastic generalization of classical common fixed point theorems. The machinery of random fixed point theory provides a convenient way of modeling many problems arising from economic theory (see e.g. [19]) and references mentioned therein. Random methods have revolutionized the financial markets. The survey article by Bharucha-Reid [7] attracted the attention of several

2010 Mathematics Subject Classification: 47H10, 54H25

Key words and phrases: cone random metric space, common random fixed point, generalized contractive condition, cone 
mathematicians and gave wings to the theory. Itoh [14] extended Spacek's and Hans's theorem to multivalued contraction mappings. Now this theory has become the full fledged research area and various ideas associated with random fixed point theory are used to obtain the solution of nonlinear random system (see $[4,5,6,11,22])$. Papageorgiou [17, 18], Beg [2, 3] studied common random fixed points and random coincidence points of a pair of compatible random operators and proved fixed point theorems for contractive random operators in Polish spaces.

In 2007, Huang and Zhang [12] introduced the concept of cone metric spaces and establish some fixed point theorems for contractive mappings in normal cone metric spaces. Subsequently, several other authors [1, 13, 21, 23] studied the existence of fixed points and common fixed points of mappings satisfying contractive type condition on a normal cone metric space.

In 2008, Rezapour and Hamlbarani [21] omitted the assumption of normality in cone metric space, which is a milestone in developing fixed point theory in cone metric space. Recently, Mehta et al. [16] introduced the concept of cone random metric space and proved an existence of random fixed point under weak contraction condition in the setting of cone random metric spaces.

In this paper, we establish some common random fixed point theorems for contractive type conditions in the setting of cone random metric spaces. Our results extend the corresponding results of [16] and some others from the current existing literature.

\section{Preliminaries}

Definition 1 (See [16]) Let $(\mathrm{E}, \tau)$ be a topological vector space. A subset $\mathrm{P}$ of $\mathrm{E}$ is called a cone whenever the following conditions hold:

$\left(\mathbf{c}_{1}\right) \mathrm{P}$ is closed, nonempty and $\mathrm{P} \neq\{0\}$;

$\left(\mathbf{c}_{2}\right) \mathrm{a}, \mathrm{b} \in \mathrm{R}, \mathrm{a}, \mathrm{b} \geq 0$ and $\mathrm{x}, \mathrm{y} \in \mathrm{P}$ imply $\mathrm{ax}+\mathrm{by} \in \mathrm{P}$;

(c) If $\mathrm{x} \in \mathrm{P}$ and $-\mathrm{x} \in \mathrm{P}$ implies $\mathrm{x}=0$.

For a given cone $\mathrm{P} \subset \mathrm{E}$, we define a partial ordering $\leq$ with respect to $\mathrm{P}$ by $x \leq y$ if and only if $y-x \in P$. We shall write $x<y$ to indicate that $x \leq y$ but $x \neq y$, while $x \ll y$ will stand for $y-x \in P^{0}$, where $P^{0}$ stands for the interior of $P$.

Definition 2 (See $[12,25])$ Let $\mathrm{X}$ be a nonempty set. Suppose that the mapping $\mathrm{d}: \mathrm{X} \times \mathrm{X} \rightarrow \mathrm{E}$ satisfies:

$\left(\mathbf{d}_{1}\right) 0 \leq \mathrm{d}(\mathrm{x}, \mathrm{y})$ for all $\mathrm{x}, \mathrm{y} \in \mathrm{X}$ and $\mathrm{d}(\mathrm{x}, \mathrm{y})=0$ if and only if $\mathrm{x}=\mathrm{y}$; 
$\left(\mathbf{d}_{\mathbf{2}}\right) \mathrm{d}(x, y)=\mathrm{d}(\mathrm{y}, \mathrm{x})$ for all $\mathrm{x}, \mathrm{y} \in \mathrm{X}$;

$\left(\mathbf{d}_{\mathbf{3}}\right) \mathrm{d}(\mathrm{x}, \mathrm{y}) \leq \mathrm{d}(\mathrm{x}, z)+\mathrm{d}(z, y)$ for all $\mathrm{x}, \mathrm{y}, z \in \mathrm{X}$.

Then $\mathrm{d}$ is called a cone metric [12] or $\mathrm{K}-m e t r i c ~[25]$ on $\mathrm{X}$ and $(\mathrm{X}, \mathrm{d})$ is called a cone metric space [12].

The concept of a cone metric space is more general than that of a metric space, because each metric space is a cone metric space where $E=\mathbb{R}$ and $\mathrm{P}=[0,+\infty)$.

Example 1 (See [12]) Let $E=\mathbb{R}^{2}, P=\left\{(x, y) \in \mathbb{R}^{2}: x \geq 0, y \geq 0\right\}, X=\mathbb{R}$ and $\mathrm{d}: \mathrm{X} \times \mathrm{X} \rightarrow \mathrm{E}$ defined by $\mathrm{d}(\mathrm{x}, \mathrm{y})=(|\mathrm{x}-\mathrm{y}|, \alpha|\mathrm{x}-\mathrm{y}|)$, where $\alpha \geq 0$ is a constant. Then $(\mathrm{X}, \mathrm{d})$ is a cone metric space with normal cone $\mathrm{P}$ where $\mathrm{K}=1$.

Example 2 (See [20]) Let $\mathrm{E}=\ell^{2}, \mathrm{P}=\left\{\left\{x_{\mathfrak{n}}\right\}_{\mathfrak{n} \geq 1} \in \mathrm{E}: \mathrm{x}_{\mathfrak{n}} \geq 0\right.$, for all $\left.\mathrm{n}\right\},(\mathrm{X}, \rho)$ a metric space, and $\mathrm{d}: \mathrm{X} \times \mathrm{X} \rightarrow \mathrm{E}$ defined by $\mathrm{d}(\mathrm{x}, \mathrm{y})=\left\{\rho(x, y) / 2^{\mathfrak{n}}\right\}_{\mathfrak{n} \geq 1}$. Then $(\mathrm{X}, \mathrm{d})$ is a cone metric space.

Clearly, the above examples show that the class of cone metric spaces contains the class of metric spaces.

Definition 3 (See [12]) Let $(\mathrm{X}, \mathrm{d})$ be a cone metric space. We say that $\left\{\mathrm{x}_{\mathrm{n}}\right\}$ is:

(i) a Cauchy sequence if for every $\varepsilon$ in $\mathrm{E}$ with $0 \ll \varepsilon$, then there is an $\mathrm{N}$ such that for all $\mathrm{n}, \mathrm{m}>\mathrm{N}, \mathrm{d}\left(\mathrm{x}_{\mathrm{n}}, \mathrm{x}_{\mathrm{m}}\right) \ll \varepsilon$;

(ii) a convergent sequence if for every $\varepsilon$ in $\mathrm{E}$ with $0 \ll \varepsilon$, then there is an $\mathrm{N}$ such that for all $\mathrm{n}>\mathrm{N}, \mathrm{d}\left(\mathrm{x}_{\mathrm{n}}, \mathrm{x}\right) \ll \varepsilon$ for some fixed $\mathrm{x}$ in $\mathrm{X}$.

A cone metric space $X$ is said to be complete if every Cauchy sequence in $X$ is convergent in $X$.

In the following $(X, d)$ will stands for a cone metric space with respect to a cone $P$ with $P^{0} \neq \emptyset$ in a real Banach space $E$ and $\leq$ is partial ordering in $E$ with respect to $P$.

Definition 4 (Measurable function) (See [16]) Let $(\Omega, \Sigma)$ be a measurable space with $\Sigma$-a sigma algebra of subsets of $\Omega$ and $M$ be a nonempty subset of a metric space $\mathrm{X}=(\mathrm{X}, \mathrm{d})$. Let $2^{\mathrm{M}}$ be the family of nonempty subsets of $\mathrm{M}$ and $\mathrm{C}(\mathrm{M})$ the family of all nonempty closed subsets of $\mathrm{M}$. A mapping $\mathrm{G}: \Omega \rightarrow 2^{\mathrm{M}}$ is called measurable if for each open subset $\mathrm{U}$ of $\mathrm{M}, \mathrm{G}^{-1}(\mathrm{U}) \in \Sigma$, where $\mathrm{G}^{-1}(\mathrm{U})=\{\omega \in \Omega: \mathrm{G}(\omega) \cap \mathrm{U} \neq \emptyset\}$. 
Definition 5 (Measurable selector) (See [16]) A mapping $\xi: \Omega \rightarrow M$ is called a measurable selector of a measurable mapping $\mathrm{G}: \Omega \rightarrow 2^{\mathrm{M}}$ if $\xi$ is measurable and $\xi(\omega) \in \mathrm{G}(\omega)$ for each $\omega \in \Omega$.

Definition 6 (Random operator) (See [16]) The mapping T: $\Omega \times M \rightarrow X$ is said to be a random operator if and only if for each fixed $x \in M$, the mapping $\mathrm{T}(., \mathrm{x}): \Omega \rightarrow \mathrm{X}$ is measurable.

Definition 7 (Continuous random operator) (See [16]) A random operator $\mathrm{T}: \Omega \times \mathrm{M} \rightarrow \mathrm{X}$ is said to be continuous random operator if for each fixed $x \in M$ and $\omega \in \Omega$, the mapping $T(\omega,):. M \rightarrow X$ is continuous.

Definition 8 (Random fixed point) (See [16]) A measurable mapping $\xi: \Omega$ $\rightarrow M$ is a random fixed point of a random operator $\mathrm{T}: \Omega \times M \rightarrow X$ if and only if $\mathrm{T}(\omega, \xi(\omega))=\xi(\omega)$ for each $\omega \in \Omega$.

Definition 9 (Cone Random Metric Space) Let $M$ be a nonempty set and let the mapping $\mathrm{d}: \Omega \times \mathrm{M} \rightarrow \mathrm{P}$, where $\mathrm{P}$ is a cone, $\omega \in \Omega$ be a selector, satisfy the following conditions:

(i) $d(x(\omega), y(\omega)) \geq 0$ and $d(x(\omega), y(\omega))=0$ if and only if $x(\omega)=y(\omega)$ for all $x(\omega), y(\omega) \in \Omega \times M$,

(ii) $d(x(\omega), y(\omega))=d(y(\omega), x(\omega))$ for all $x, y \in M, \omega \in \Omega$ and $x(\omega), y(\omega)$ $\in \Omega \times M$,

(iii) $d(x(\omega), y(\omega)) \leq d(x(\omega), z(\omega))+d(z(\omega), y(\omega))$ for all $x, y, z \in M$ and $\omega \in \Omega$ be a selector,

(iv) for any $x, y \in M, \omega \in \Omega, d(x(\omega), y(\omega))$ is non-increasing and left continuous.

Then $\mathrm{d}$ is called cone random metric on $\mathrm{M}$ and $(\mathrm{M}, \mathrm{d})$ is called a cone random metric space.

Definition 10 Let $(\mathrm{X}, \mathrm{d})$ be a metric space. A mapping $\mathrm{T}: \mathrm{X} \rightarrow \mathrm{X}$ is called an a-contraction if

$$
d(T x, T y) \leq a d(x, y) \text { for all } x, y \in X,
$$

where $a \in(0,1)$. 
Definition 11 The mapping $\mathrm{T}$ is called Kannan contraction mapping [15] if there exists $\mathrm{b} \in\left(0, \frac{1}{2}\right)$ such that

$$
d(T x, T y) \leq b[d(x, T x)+d(y, T y)] \text { for all } x, y \in X .
$$

Definition 12 The mapping $\mathrm{T}$ is called Chatterjea contraction mapping [8] if there exists $\mathrm{c} \in\left(0, \frac{1}{2}\right)$ such that

$$
d(T x, T y) \leq c[d(x, T y)+d(y, T x)] \text { for all } x, y \in X
$$

\section{Generalized contraction condition for two mappings}

Let $(X, d)$ be a metric space and let $S, T: X \rightarrow X$ be two mappings satisfying the condition:

$$
\begin{aligned}
d(S x, T y) \leq & a d(x, y)+b[d(x, S x)+d(y, T y)] \\
& +c[d(x, T y)+d(y, S x)]
\end{aligned}
$$

for all $x, y \in X$ and $a+2 b+2 c<1$, where $a, b, c>0$ are constants.

Remark 1 (i) If we take $\mathrm{S}=\mathrm{T}$ and $\mathrm{b}=\mathrm{c}=0$, then condition (4) reduces to the contraction condition (1).

(ii) If we take $\mathrm{S}=\mathrm{T}$ and $\mathrm{a}=\mathrm{c}=0$, then condition (4) reduces to the Kannan contraction condition (2).

(iii) If we take $\mathrm{S}=\mathrm{T}$ and $\mathrm{a}=\mathrm{b}=0$, then condition (4) reduces to the Chatterjea contraction condition (3).

Thus it is clear from Remark 1 that the generalized contraction condition for one or two mappings is weaker than Banach contraction, Kannan contraction and Chatterjea contraction conditions.

\section{Main results}

In this section we shall prove some common random fixed point theorems under generalized contractive condition (4) in the setting of cone random metric spaces.

Theorem 1 Let $(\mathrm{X}, \mathrm{d})$ be a complete cone random metric space with respect to a cone $\mathrm{P}$ and let $\mathrm{M}$ be a nonempty separable closed subset of $\mathrm{X}$. Let $\mathrm{S}$ and 
$\mathrm{T}$ be two continuous random operators defined on $M$ such that for $\omega \in \Omega$, $S(\omega,),. T(\omega,):. \Omega \times M \rightarrow M$ satisfying the condition:

$$
\begin{aligned}
d(S(x(\omega)), T(y(\omega))) \leq & a(\omega) d(x(\omega), y(\omega)) \\
& +b(\omega)[d(x(\omega), S(x(\omega)))+d(y(\omega), T(y(\omega)))] \\
& +c(\omega)[d(x(\omega), T(y(\omega)))+d(y(\omega), S(x(\omega)))]
\end{aligned}
$$

for all $x, y \in M, a(\omega)+2 b(\omega)+2 c(\omega)<1$, where $a(\omega), b(\omega), c(\omega)>0$ and $\omega \in \Omega$. Then $\mathrm{S}$ and $\mathrm{T}$ have a unique common random fixed point in $\mathrm{X}$.

Proof. For each $x_{0}(\omega) \in \Omega \times M$ and $n=0,1,2, \ldots$, we choose $x_{1}(\omega), x_{2}(\omega) \in$ $\Omega \times M$ such that $x_{1}(\omega)=S\left(x_{0}(\omega)\right)$ and $x_{2}(\omega)=T\left(x_{1}(\omega)\right)$. In general we define sequence of elements of $M$ such that $x_{2 n+1}(\omega)=S\left(x_{2 n}(\omega)\right)$ and $x_{2 n+2}(\omega)=$ $\mathrm{T}\left(\mathrm{x}_{2 \mathrm{n}+1}(\omega)\right)$. Then from (5), we have

$$
\begin{aligned}
d\left(x_{2 n+1}(\omega), x_{2 n}(\omega)\right)= & d\left(S\left(x_{2 n}(\omega)\right), T\left(x_{2 n-1}(\omega)\right)\right) \\
\leq & a(\omega) d\left(x_{2 n}(\omega), x_{2 n-1}(\omega)\right)+b(\omega)\left[d\left(x_{2 n}(\omega), S\left(x_{2 n}(\omega)\right)\right)\right. \\
& \left.+d\left(x_{2 n-1}(\omega), T\left(x_{2 n-1}(\omega)\right)\right)\right] \\
& +c(\omega)\left[d\left(x_{2 n}(\omega), T\left(x_{2 n-1}(\omega)\right)\right)\right. \\
& +d\left(x_{2 n-1}(\omega), S\left(x_{2 n}(\omega)\right)\right] \\
\leq & a(\omega) d\left(x_{2 n}(\omega), x_{2 n-1}(\omega)\right)+b(\omega)\left[d\left(x_{2 n}(\omega), x_{2 n+1}(\omega)\right)\right. \\
& \left.+d\left(x_{2 n-1}(\omega), x_{2 n}(\omega)\right)\right]+c(\omega)\left[d\left(x_{2 n}(\omega), x_{2 n}(\omega)\right)\right. \\
& \left.+d\left(x_{2 n-1}(\omega), x_{2 n+1}(\omega)\right)\right] \\
= & a(\omega) d\left(x_{2 n}(\omega), x_{2 n-1}(\omega)\right)+b(\omega)\left[d\left(x_{2 n}(\omega), x_{2 n+1}(\omega)\right)\right. \\
& \left.+d\left(x_{2 n-1}(\omega), x_{2 n}(\omega)\right)\right] c c(\omega) d\left(x_{2 n-1}(\omega), x_{2 n+1}(\omega)\right) \\
\leq & a(\omega) d\left(x_{2 n}(\omega), x_{2 n-1}(\omega)\right)+b(\omega)\left[d\left(x_{2 n}(\omega), x_{2 n+1}(\omega)\right)\right. \\
& \left.+d\left(x_{2 n-1}(\omega), x_{2 n}(\omega)\right)\right]+c(\omega)\left[d\left(x_{2 n-1}(\omega), x_{2 n}(\omega)\right)\right. \\
& \left.+d\left(x_{2 n}(\omega), x_{2 n+1}(\omega)\right)\right] \\
= & (a(\omega)+b(\omega)+c(\omega)) d\left(x_{2 n}(\omega), x_{2 n-1}(\omega)\right) \\
& +(b(\omega)+c(\omega)) d\left(x_{2 n+1}(\omega), x_{2 n}(\omega)\right)
\end{aligned}
$$

Therefore,

$$
\begin{aligned}
d\left(x_{2 n+1}(\omega), x_{2 n}(\omega)\right) & \leq\left(\frac{a(\omega)+b(\omega)+c(\omega)}{1-b(\omega)-c(\omega)}\right) d\left(x_{2 n}(\omega), x_{2 n-1}(\omega)\right) \\
& =\lambda d\left(x_{2 n}(\omega), x_{2 n-1}(\omega)\right),
\end{aligned}
$$


where

$$
\lambda=\left(\frac{a(\omega)+b(\omega)+c(\omega)}{1-b(\omega)-c(\omega)}\right) .
$$

By the assumption of the theorem

$$
\begin{aligned}
a(\omega)+2 b(\omega)+2 c(\omega)<1 & \Rightarrow a(\omega)+b(\omega)+c(\omega)<1-b(\omega)-c(\omega) \\
& \Rightarrow \lambda=\left(\frac{a(\omega)+b(\omega)+c(\omega)}{1-b(\omega)-c(\omega)}\right)<1 .
\end{aligned}
$$

Similarly, we have

$$
d\left(x_{2 n}(\omega), x_{2 n-1}(\omega)\right) \leq \lambda d\left(x_{2 n-1}(\omega), x_{2 n-2}(\omega)\right) .
$$

Hence

$$
d\left(x_{2 n+1}(\omega), x_{2 n}(\omega)\right) \leq \lambda^{2} d\left(x_{2 n-1}(\omega), x_{2 n-2}(\omega)\right) .
$$

On continuing this process, we get

$$
d\left(x_{2 n+1}(\omega), x_{2 n}(\omega)\right) \leq \lambda^{2 n} d\left(x_{1}(\omega), x_{0}(\omega)\right) .
$$

Also for $n>m$, we have

$$
\begin{aligned}
d\left(x_{n}(\omega), x_{m}(\omega)\right) \leq & d\left(x_{n}(\omega), x_{n-1}(\omega)\right)+d\left(x_{n-1}(\omega), x_{n-2}(\omega)\right)+\ldots \\
& +d\left(x_{m+1}(\omega), x_{m}(\omega)\right) \\
\leq & \left(\lambda^{n-1}+\lambda^{n-2}+\cdots+\lambda^{m}\right) d\left(x_{1}(\omega), x_{0}(\omega)\right) \\
\leq & \left(\frac{\lambda^{m}}{1-\lambda}\right) d\left(x_{1}(\omega), x_{0}(\omega)\right) .
\end{aligned}
$$

Let $0 \ll \varepsilon$ be given. Choose a natural number $N$ such that $\left(\frac{\lambda^{m}}{1-\lambda}\right) d\left(x_{1}(\omega), x_{0}(\omega)\right)$ $\ll \varepsilon$ for every $m \geq N$. Thus

$$
\mathrm{d}\left(x_{\mathfrak{n}}(\omega), x_{\mathrm{m}}(\omega)\right) \leq\left(\frac{\lambda^{\mathrm{m}}}{1-\lambda}\right) \mathrm{d}\left(x_{1}(\omega), x_{0}(\omega)\right) \ll \varepsilon,
$$

for every $n>m \geq N$. This shows that the sequence $\left\{x_{n}(\omega)\right\}$ is a Cauchy sequence in $\Omega \times M$. Since $(X, d)$ is complete, there exists $z(\omega) \in \Omega \times X$ such that $x_{n}(\omega) \rightarrow z(\omega)$ as $n \rightarrow \infty$. Choose a natural number $N_{1}$ such that

$$
d\left(x_{2 n+1}(\omega), x_{2 n+2}(\omega)\right) \ll \frac{\varepsilon(1-b(\omega)-c(\omega))}{2(a(\omega)+b(\omega)+c(\omega))},
$$


and

$$
d\left(z(\omega), x_{2 n+2}(\omega)\right) \ll \frac{\varepsilon(1-b(\omega)-c(\omega))}{2(1+a(\omega)+2 c(\omega))},
$$

for every $n \geq N_{1}$. Hence for $n \geq N_{1}$, we have

$$
\begin{aligned}
d(z(\omega), S(z(\omega))) \leq & d\left(z(\omega), x_{2 n+2}(\omega)\right)+d\left(x_{2 n+2}(\omega), S(z(\omega))\right. \\
= & d\left(z(\omega), x_{2 n+2}(\omega)\right)+d\left(S \left(z(\omega), T\left(x_{2 n+1}(\omega)\right)\right.\right. \\
\leq & d\left(z(\omega), x_{2 n+2}(\omega)\right)+a(\omega) d\left(z(\omega), x_{2 n+1}(\omega)\right) \\
& +b(\omega)\left[d(z(\omega), S(z(\omega)))+d\left(x_{2 n+1}(\omega), T\left(x_{2 n+1}(\omega)\right)\right)\right] \\
& +c(\omega)\left[d\left(z(\omega), T\left(x_{2 n+1}(\omega)\right)\right)+d\left(x_{2 n+1}(\omega), S(z(\omega))\right)\right] \\
= & d\left(z(\omega), x_{2 n+2}(\omega)\right)+a(\omega) d\left(z(\omega), x_{2 n+1}(\omega)\right) \\
& +b(\omega)\left[d(z(\omega), S(z(\omega)))+d\left(x_{2 n+1}(\omega), x_{2 n+2}(\omega)\right)\right] \\
& +c(\omega)\left[d\left(z(\omega), x_{2 n+2}(\omega)\right)+d\left(x_{2 n+1}(\omega), S(z(\omega))\right)\right] \\
\leq & d\left(z(\omega), x_{2 n+2}(\omega)\right) \\
& +a(\omega)\left[d\left(z(\omega), x_{2 n+2}(\omega)\right)+d\left(x_{2 n+2}(\omega), x_{2 n+1}(\omega)\right)\right] \\
& +b(\omega)\left[d(z(\omega), S(z(\omega)))+d\left(x_{2 n+1}(\omega), x_{2 n+2}(\omega)\right)\right] \\
& +c(\omega)\left[d\left(z(\omega), x_{2 n+2}(\omega)\right)+d\left(x_{2 n+1}(\omega), x_{2 n+2}(\omega)\right)\right. \\
& \left.+d\left(x_{2 n+2}(\omega), z(\omega)\right)+d(z(\omega), S(z(\omega)))\right] \\
= & (1+a(\omega)+2 c(\omega)) d\left(z(\omega), x_{2 n+2}(\omega)\right) \\
& +(b(\omega)+c(\omega)) d(z(\omega), S(z(\omega))) \\
& +(a(\omega)+b(\omega)+c(\omega)) d\left(x_{2 n+1}(\omega), x_{2 n+2}(\omega)\right) .
\end{aligned}
$$

The above inequality gives

$$
\begin{aligned}
d(z(\omega), S(z(\omega))) \leq & \left(\frac{1+a(\omega)+2 c(\omega)}{1-b(\omega)-c(\omega)}\right) d\left(z(\omega), x_{2 n+2}(\omega)\right) \\
& +\left(\frac{a(\omega)+b(\omega)+c(\omega)}{1-b(\omega)-c(\omega)}\right) d\left(x_{2 n+1}(\omega), x_{2 n+2}(\omega)\right) .
\end{aligned}
$$

Using (7) and (8) in (9), we get

$$
\mathrm{d}(z(\omega), S(z(\omega))) \ll \frac{\varepsilon}{2}+\frac{\varepsilon}{2}=\varepsilon .
$$

Thus $d(z(\omega), S(z(\omega))) \ll \frac{\varepsilon}{m}$ for all $m \geq 1$. So $\frac{\varepsilon}{m}-d(z(\omega), S(z(\omega))) \in P$ for all $\mathrm{m} \geq 1$. Since $\frac{\varepsilon}{\mathrm{m}} \rightarrow 0$ as $\mathrm{m} \rightarrow \infty$ and $\mathrm{P}$ is closed, $-\mathrm{d}(z(\omega), S(z(\omega))) \in \mathrm{P}$. 
But $d(z(\omega), S(z(\omega))) \in$ P. Therefore by definition $\mathbf{1}\left(\mathbf{c}_{\boldsymbol{3}}\right), \mathrm{d}(z(\omega), \mathrm{S}(z(\omega))$ $=0$ and so $S(z(\omega))=z(\omega)$.

In an exactly the similar way we can prove that for all $\omega \in \Omega, \mathrm{T}(z(\omega))=$ $z(\omega)$. Hence $S(z(\omega))=T(z(\omega))=z(\omega)$. This shows that $z(\omega)$ is a common random fixed point of $S$ and $T$.

\section{Uniqueness}

Let $v(\omega)$ be another random fixed point common to $S$ and $T$, that is, for $\omega \in \Omega, S(v(\omega))=T(v(\omega))=v(\omega)$. Then for $\omega \in \Omega$, we have

$$
\begin{aligned}
\mathrm{d}(z(\omega), v(\omega))= & d(S(z(\omega)), \mathrm{T}(v(\omega))) \\
\leq & \mathrm{a}(\omega) \mathrm{d}(z(\omega), v(\omega))+\mathrm{b}(\omega)[\mathrm{d}(z(\omega), S(z(\omega))) \\
& +\mathrm{d}(v(\omega), \mathrm{T}(v(\omega)))]+c(\omega)[d(z(\omega), \mathrm{T}(v(\omega)) \\
& +d(v(\omega), S(z(\omega)))] \\
\leq & (a(\omega)+2 c(\omega)) d(z(\omega), v(\omega)) \\
< & d(z(\omega), v(\omega)), \text { since } 0<a(\omega)+2 c(\omega)<1,
\end{aligned}
$$

a contradiction. Hence $z(\omega)=v(\omega)$ and so $z(\omega)$ is a unique common random fixed point of $S$ and $T$. This completes the proof.

Corollary 1 Let $(\mathrm{X}, \mathrm{d})$ be a complete cone random metric space with respect to a cone $\mathrm{P}$ and let $\mathrm{M}$ be a nonempty separable closed subset of $\mathrm{X}$. Let $\mathrm{T}$ be a continuous random operator defined on $M$ such that for $\omega \in \Omega, T(\omega,):. \Omega \times$ $\mathrm{M} \rightarrow \mathrm{M}$ satisfying the condition:

$$
\begin{aligned}
d(T(x(\omega)), T(y(\omega))) \leq & a(\omega) d(x(\omega), y(\omega)) \\
& +b(\omega)[d(x(\omega), T(x(\omega)))+d(y(\omega), T(y(\omega)))] \\
& +c(\omega)[d(x(\omega), T(y(\omega)))+d(y(\omega), T(x(\omega)))]
\end{aligned}
$$

for all $x, y \in M, a(\omega)+2 b(\omega)+2 c(\omega)<1$, where $a(\omega), b(\omega), c(\omega)>0$ and $\omega \in \Omega$. Then $\mathrm{T}$ has a unique random fixed point in $\mathrm{X}$.

Proof. The proof of the corollary immediately follows by putting $S=T$ in Theorem 1. This completes the proof.

If we take $S=T$ and $b(\omega)=c(\omega)=0$ in Theorem 1 , then we obtain the following result as corollary. 
Corollary 2 Let $(\mathrm{X}, \mathrm{d})$ be a complete cone random metric space with respect to a cone $\mathrm{P}$ and let $\mathrm{M}$ be a nonempty separable closed subset of $\mathrm{X}$. Let $\mathrm{T}$ be a random operator defined on $M$ such that for $\omega \in \Omega, T(\omega,):. \Omega \times M \rightarrow M$ satisfying the condition:

$$
d(T(x(\omega)), T(y(\omega))) \leq a(\omega) d(x(\omega), y(\omega)),
$$

for all $x, y \in M, a(\omega) \in(0,1)$ and $\omega \in \Omega$. Then $T$ has a unique random fixed point in $\mathrm{X}$.

If we take $S=T$ and $a(\omega)=c(\omega)=0$ in Theorem 1, then we obtain the following result as corollary.

Corollary 3 ([16], Corollary 3.2) Let (X, d) be a complete cone random metric space with respect to a cone $\mathrm{P}$ and let $\mathrm{M}$ be a nonempty separable closed subset of $\mathrm{X}$. Let $\mathrm{T}$ be a continuous random operator defined on $\mathrm{M}$ such that for $\omega \in \Omega, T(\omega,):. \Omega \times M \rightarrow M$ satisfying the condition:

$$
\mathrm{d}(\mathrm{T}(\mathrm{x}(\omega)), \mathrm{T}(\mathrm{y}(\omega))) \leq \mathrm{b}(\omega)[\mathrm{d}(\mathrm{x}(\boldsymbol{\omega}), \mathrm{T}(\mathrm{x}(\boldsymbol{\omega})))+\mathrm{d}(\mathrm{y}(\omega), \mathrm{T}(\mathrm{y}(\omega)))]
$$

for all $\mathrm{x}, \mathrm{y} \in \mathrm{M}, \mathrm{b}(\omega) \in\left(0, \frac{1}{2}\right)$ and $\omega \in \Omega$. Then $\mathrm{T}$ has a unique random fixed point in $\mathrm{X}$.

If we take $S=T$ and $a(\omega)=b(\omega)=0$ in Theorem 1, then we obtain the following result as corollary.

Corollary 4 ([16], Corollary 3.3) Let (X, d) be a complete cone random metric space with respect to a cone $\mathrm{P}$ and let $\mathrm{M}$ be a nonempty separable closed subset of $\mathrm{X}$. Let $\mathrm{T}$ be a continuous random operator defined on $\mathrm{M}$ such that for $\omega \in \Omega, T(\omega,):. \Omega \times M \rightarrow M$ satisfying the condition:

$$
\mathrm{d}(\mathrm{T}(\mathrm{x}(\omega)), \mathrm{T}(\mathrm{y}(\omega))) \leq \mathrm{c}(\omega)[\mathrm{d}(\mathrm{x}(\omega), \mathrm{T}(\mathrm{y}(\omega)))+\mathrm{d}(\mathrm{y}(\omega), \mathrm{T}(\mathrm{x}(\omega)))]
$$

for all $\mathrm{x}, \mathrm{y} \in \mathrm{M}, \mathrm{c}(\omega) \in\left(0, \frac{1}{2}\right)$ and $\omega \in \Omega$. Then $\mathrm{T}$ has a unique random fixed point in $\mathrm{X}$.

Theorem 2 Let $(\mathrm{X}, \mathrm{d})$ be a complete cone random metric space with respect to a cone $\mathrm{P}$ and let $\mathrm{M}$ be a nonempty separable closed subset of $\mathrm{X}$. Let $\mathrm{S}$ and $\mathrm{T}$ be two continuous random operators defined on $M$ such that for $\omega \in \Omega$, $S(\omega,),. T(\omega,):. \Omega \times M \rightarrow M$ satisfying the condition:

$$
\begin{aligned}
d(S(x(\omega)), T(y(\omega))) \leq h(\omega) & \max \{d(x(\omega), y(\omega)), d(x(\omega), S(x(\omega))), \\
& d(y(\omega), T(y(\omega))), d(x(\omega), T(y(\omega))), \\
& d(y(\omega), S(x(\omega)))\}
\end{aligned}
$$


for all $x, y \in M, 0<h(\omega)<1$ and $\omega \in \Omega$. Then $S$ and $T$ have a unique common random fixed point in $\mathrm{X}$.

Proof. For each $x_{0}(\omega) \in \Omega \times M$ and $n=0,1,2, \ldots$, we choose $x_{1}(\omega), x_{2}(\omega)$ $\in \Omega \times M$ such that $x_{1}(\omega)=S\left(x_{0}(\omega)\right)$ and $x_{2}(\omega)=T\left(x_{1}(\omega)\right)$. In general we define sequence of elements of $M$ such that $x_{2 n+1}(\omega)=S\left(x_{2 n}(\omega)\right)$ and $x_{2 n+2}(\omega)=T\left(x_{2 n+1}(\omega)\right)$. Then from (11), we have

$$
\begin{aligned}
d\left(x_{2 n+1}(\omega), x_{2 n}(\omega)\right)= & d\left(S\left(x_{2 n}(\omega)\right), T\left(x_{2 n-1}(\omega)\right)\right) \\
\leq & h(\omega) \max \left\{d\left(x_{2 n}(\omega), x_{2 n-1}(\omega)\right),\right. \\
& d\left(x_{2 n}(\omega), S\left(x_{2 n}(\omega)\right)\right), d\left(x_{2 n-1}(\omega), T\left(x_{2 n-1}(\omega)\right)\right), \\
& \left.d\left(x_{2 n}(\omega), T\left(x_{2 n-1}(\omega)\right)\right), d\left(x_{2 n-1}(\omega), S\left(x_{2 n}(\omega)\right)\right)\right\} \\
= & h(\omega) \max \left\{d\left(x_{2 n}(\omega), x_{2 n-1}(\omega)\right),\right. \\
& d\left(x_{2 n}(\omega), x_{2 n+1}(\omega)\right), d\left(x_{2 n-1}(\omega), x_{2 n}(\omega)\right), \\
& \left.d\left(x_{2 n}(\omega), x_{2 n}(\omega)\right), d\left(x_{2 n-1}(\omega), x_{2 n+1}(\omega)\right)\right\} \\
= & h(\omega) \max \left\{d\left(x_{2 n}(\omega), x_{2 n-1}(\omega)\right),\right. \\
& d\left(x_{2 n}(\omega), x_{2 n+1}(\omega)\right), d\left(x_{2 n-1}(\omega), x_{2 n}(\omega)\right), \\
& \left.d\left(x_{2 n-1}(\omega), x_{2 n+1}(\omega)\right)\right\} \\
\leq & h(\omega) d\left(x_{2 n}(\omega), x_{2 n-1}(\omega)\right) .
\end{aligned}
$$

Similarly, we have

$$
d\left(x_{2 n}(\omega), x_{2 n-1}(\omega)\right) \leq h(\omega) d\left(x_{2 n-1}(\omega), x_{2 n-2}(\omega)\right) .
$$

Hence

$$
d\left(x_{2 n+1}(\omega), x_{2 n}(\omega)\right) \leq h(\omega)^{2} d\left(x_{2 n-1}(\omega), x_{2 n-2}(\omega)\right) .
$$

On continuing this process, we get

$$
d\left(x_{2 n+1}(\omega), x_{2 n}(\omega)\right) \leq h(\omega)^{2 n} d\left(x_{1}(\omega), x_{0}(\omega)\right) .
$$

Also for $n>m$, we have

$$
\begin{aligned}
d\left(x_{n}(\omega), x_{m}(\omega)\right) \leq & d\left(x_{n}(\omega), x_{n-1}(\omega)\right)+d\left(x_{n-1}(\omega), x_{n-2}(\omega)\right)+\ldots \\
& +d\left(x_{m+1}(\omega), x_{m}(\omega)\right) \\
\leq & \left(h(\omega)^{n-1}+h(\omega)^{n-2}+\cdots+h(\omega)^{m}\right) d\left(x_{1}(\omega), x_{0}(\omega)\right)
\end{aligned}
$$




$$
\leq\left(\frac{h(\omega)^{m}}{1-h(\omega)}\right) d\left(x_{1}(\omega), x_{0}(\omega)\right)
$$

Let $0 \ll \varepsilon$ be given. Choose a natural number $N$ such that $\left(\frac{h(\omega)^{m}}{1-h(\omega)}\right) d\left(x_{1}(\omega)\right.$, $\left.x_{0}(\omega)\right) \ll \varepsilon$ for every $m \geq N$. Thus

$$
d\left(x_{n}(\omega), x_{m}(\omega)\right) \leq\left(\frac{h(\omega)^{m}}{1-h(\omega)}\right) d\left(x_{1}(\omega), x_{0}(\omega)\right) \ll \varepsilon,
$$

for every $n>m \geq N$.

This shows that the sequence $\left\{x_{n}(\omega)\right\}$ is a Cauchy sequence in $\Omega \times M$. Since $(X, d)$ is complete, there exists $z(\omega) \in \Omega \times X$ such that $x_{n}(\omega) \rightarrow z(\omega)$ as $\mathrm{n} \rightarrow \infty$. Hence, we have

$$
\begin{aligned}
d(z(\omega), S(z(\omega))) \leq & d\left(z(\omega), x_{2 n+2}(\omega)\right)+d\left(x_{2 n+2}(\omega), S(z(\omega))\right. \\
= & d\left(z(\omega), x_{2 n+2}(\omega)\right)+d\left(S(z(\omega)), T\left(x_{2 n+1}(\omega)\right)\right) \\
\leq & d\left(z(\omega), x_{2 n+2}(\omega)\right)+h(\omega) \max \left\{d\left(z(\omega), x_{2 n+1}(\omega)\right),\right. \\
& d(z(\omega), S(z(\omega))), d\left(x_{2 n+1}(\omega), T\left(x_{2 n+1}(\omega)\right)\right), \\
& \left.d\left(z(\omega), T\left(x_{2 n+1}(\omega)\right)\right), d\left(x_{2 n+1}(\omega), S(z(\omega))\right)\right\} \\
= & d\left(z(\omega), x_{2 n+2}(\omega)\right)+h(\omega) \max \left\{d\left(z(\omega), x_{2 n+1}(\omega)\right),\right. \\
& d(z(\omega), S(z(\omega))), d\left(x_{2 n+1}(\omega), x_{2 n+2}(\omega)\right), \\
& \left.d\left(z(\omega), x_{2 n+2}(\omega)\right), d\left(x_{2 n+1}(\omega), S(z(\omega))\right)\right\}
\end{aligned}
$$

Taking the limit as $\mathrm{n} \rightarrow \infty$ in the above inequality, we get

$$
d(z(\omega), S(z(\omega))) \leq h(\omega) d(z(\omega), S(z(\omega)))
$$

or,

$$
\begin{gathered}
(1-h(\omega)) d(z(\omega), S(z(\omega))) \leq 0 \\
\Rightarrow d(z(\omega), S(z(\omega))) \leq 0, \text { since } 0<(1-h(\omega))<1 .
\end{gathered}
$$

Thus $-\mathrm{d}(z(\omega), S(z(\omega))) \in$ P. But $d(z(\omega), S(z(\omega))) \in$ P. Therefore by definition $\mathbf{1}\left(\mathbf{c}_{\mathbf{3}}\right)$, we have $\mathrm{d}(z(\omega), S(z(\omega))=0$ and so $S(z(\omega))=z(\omega)$.

In an exactly the similar way we can prove that for all $\omega \in \Omega, \mathrm{T}(z(\omega))=$ $z(\omega)$. Hence $S(z(\omega))=T(z(\omega))=z(\omega)$. This shows that $z(\omega)$ is a common random fixed point of $S$ and $T$. Rest of the proof is same as that of Theorem 1. This completes the proof.

If we take $S=T$ in Theorem 2 we get the following result as corollary. 
Corollary 5 Let $(\mathrm{X}, \mathrm{d})$ be a complete cone random metric space with respect to a cone $\mathrm{P}$ and let $\mathrm{M}$ be a nonempty separable closed subset of $\mathrm{X}$. Let $\mathrm{T}$ be a continuous random operators defined on $M$ such that for $\omega \in \Omega, T(\omega,):. \Omega \times$ $\mathrm{M} \rightarrow \mathrm{M}$ satisfying the condition:

$$
\begin{aligned}
d(T(x(\omega)), T(y(\omega))) \leq h(\omega) & \max \{d(x(\omega), y(\omega)), d(x(\omega), T(x(\omega))), \\
& d(y(\omega), T(y(\omega))), d(x(\omega), T(y(\omega))), \\
& d(y(\omega), T(x(\omega)))\}
\end{aligned}
$$

for all $\mathrm{x}, \mathrm{y} \in \mathrm{M}, 0<\mathrm{h}(\mathrm{\omega})<1$ and $\omega \in \Omega$. Then $\mathrm{T}$ has a unique random fixed point in $\mathrm{X}$.

Proof. The proof of corollary 5 immediately follows by putting $S=T$ in Theorem 2. This completes the proof.

The following corollary is a special case of Corollary 5 .

Corollary 6 Let $(\mathrm{X}, \mathrm{d})$ be a complete cone random metric space with respect to a cone $\mathrm{P}$ and let $\mathrm{M}$ be a nonempty separable closed subset of $\mathrm{X}$. Let $\mathrm{T}$ be a continuous random operators defined on $M$ such that for $\omega \in \Omega, T(\omega,):. \Omega \times$ $M \rightarrow M$ satisfying the condition:

$$
d(T(x(\omega)), T(y(\omega))) \leq h(\omega) d(x(\omega), y(\omega))
$$

for all $\mathrm{x}, \mathrm{y} \in \mathrm{M}, 0<\mathrm{h}(\mathrm{\omega})<1$ and $\omega \in \Omega$. Then $\mathrm{T}$ has a unique random fixed point in $\mathrm{X}$.

Condition (14) is called Banach contractive condition.

Proof. (Proof of corollary 6) The proof of corollary 6 immediately follows from Corollary 5 by taking

$$
\begin{aligned}
& \max \{d(x(\omega), y(\omega)), d(x(\omega), T(x(\omega))), d(y(\omega), T(y(\omega))), \\
& d(x(\omega), T(y(\omega))), d(y(\omega), T(x(\omega)))\}=d(x(\omega), y(\omega)) .
\end{aligned}
$$

This completes the proof.

Example 3 Let $\Omega=[0,1]$ and $\Sigma$ be the sigma algebra of Lebesgue's measurable subset of $[0,1]$. Take $\mathrm{X}=\mathrm{R}$ with $\mathrm{d}(\mathrm{x}, \mathrm{y})=|\mathrm{x}-\mathrm{y}|$ for $\mathrm{x}, \mathrm{y} \in \mathrm{R}$. Define random mapping $\mathrm{T}$ from $\Omega \times \mathrm{X}$ to $\mathrm{X}$ as $\mathrm{T}(\omega, \mathrm{x})=\omega-\mathrm{x}$. Then a measurable mapping $\xi: \Omega \rightarrow X$ defined as $\xi(\omega)=\frac{\omega}{2}$ for all $\omega \in \Omega$, serve as a unique random fixed point of $\mathrm{T}$. 
Example 4 Let $M=R$ and $P=\{x \in M: x \geq 0\}$, also $\Omega=[0,1]$ and $\Sigma$ be the sigma algebra of Lebesgue's measurable subset of $[0,1]$. Let $\mathrm{X}=[0, \infty)$ and define a mapping $\mathrm{d}:(\Omega \times \mathrm{X}) \times(\Omega \times \mathrm{X}) \rightarrow \mathrm{M}$ by $\mathrm{d}(\mathrm{x}(\omega), \mathrm{y}(\omega))=|\mathrm{x}(\omega)-\mathrm{y}(\omega)|$. Then $(\mathrm{X}, \mathrm{d})$ is a cone random metric space. Define random operator $\mathrm{T}$ form $(\Omega \times X)$ to $\mathrm{X}$ as $\mathrm{T}(\omega, \mathrm{x})=\frac{1-\omega^{2}+2 \mathrm{x}}{3}$. Also sequence of mapping $\xi_{n}: \Omega \rightarrow \mathrm{X}$ is defined by $\xi_{n}(\omega)=\left(1-\omega^{2}\right)^{1+(1 / n)}$ for every $\omega \in \Omega$ and $n \in N$. Define measurable mapping $\xi: \Omega \rightarrow X$ as $\xi(\omega)=\left(1-\omega^{2}\right)$ for every $\omega \in \Omega$. Hence $\left(1-\omega^{2}\right)$ is the random fixed point of the random operator $\mathrm{T}$.

Example 5 Let $\mathrm{M}=\mathrm{R}$ and $\mathrm{P}=\{\mathrm{x} \in \mathrm{M}: \mathrm{x} \geq 0\}$, also $\Omega=[0,1]$ and $\Sigma$ be the sigma algebra of Lebesgue's measurable subset of $[0,1]$. Let $\mathrm{X}=[0, \infty)$ and define a mapping $\mathrm{d}:(\Omega \times \mathrm{X}) \times(\Omega \times \mathrm{X}) \rightarrow \mathrm{M}$ by $\mathrm{d}(\mathrm{x}(\omega), \mathrm{y}(\omega))=|\mathrm{x}(\omega)-\mathrm{y}(\omega)|$. Then $(\mathrm{X}, \mathrm{d})$ is a cone random metric space. Define random operators $\mathrm{S}$ and $\mathrm{T}$ form $(\Omega \times \mathrm{X})$ to $\mathrm{X}$ as $\mathrm{S}(\omega, \mathrm{x})=\frac{1-\omega^{2}+\mathrm{x}}{2}$ and $\mathrm{T}(\omega, \mathrm{x})=\frac{1-\omega^{2}+2 \mathrm{x}}{3}$. Also sequence of mapping $\xi_{n}: \Omega \rightarrow X$ is defined by $\xi_{n}(\omega)=\left(1-\omega^{2}\right)^{1+(1 / n)}$ for every $\omega \in \Omega$ and $\mathrm{n} \in \mathrm{N}$. Define measurable mapping $\xi: \Omega \rightarrow \mathrm{X}$ as $\xi(\omega)=\left(1-\omega^{2}\right)$ for every $\omega \in \Omega$. Hence $\left(1-\omega^{2}\right)$ is a common random fixed point of the random operators $\mathrm{S}$ and $\mathrm{T}$.

Example 6 Let $\mathrm{E}=\{0,1,2,3,4\} \subset \mathrm{R}$ with the usual metric d. Consider $\Omega=\{0,1,2,3,4\}$ and let $\Sigma$ be the sigma algebra of Lebesgue's measurable subset of $\Omega$. Define $\mathrm{S}, \mathrm{T}: \Omega \times \mathrm{E} \rightarrow \mathrm{E}$ by

$$
\left\{\begin{aligned}
S(\omega, x) & =3, & & \text { where } x=0 \text { and } \omega \in \Omega \\
& =1, & & \text { otherwise }
\end{aligned}\right.
$$

and

$$
\left\{\begin{aligned}
\mathrm{T}(\omega, \mathrm{x}) & =2, & & \text { where } x=0 \text { and } \omega \in \Omega \\
& =1, & & \text { otherwise. }
\end{aligned}\right.
$$

Let us take $x(\omega)=0, y(\omega)=1$. Then from condition (11), we have

$$
\begin{aligned}
2 & =d(S(x(\omega)), T(y(\omega))) \\
\leq & h(\omega) \max \{d(x(\omega), y(\omega)), d(x(\omega), S(x(\omega))), \\
& d(y(\omega), T(y(\omega))), d(x(\omega), T(y(\omega))), \\
& d(y(\omega), S(x(\omega)))\} \\
& =h(\omega) \max \{1,3,0,1,2\}
\end{aligned}
$$


which implies $\mathrm{h}(\boldsymbol{\omega}) \geq \frac{2}{3}$. Now if we take $0<\mathrm{h}(\boldsymbol{\omega})<1$, then condition (11) is satisfied. The measurable function $\xi: \Omega \rightarrow \mathrm{E}$ with $\xi(\omega)=1$ is a unique common random fixed point of $\mathrm{S}$ and $\mathrm{T}$, that is, $\mathrm{S}(\omega, \mathrm{x})=\mathrm{T}(\omega, \mathrm{x})=1=\xi(\omega)$.

Example 7 Let $\mathrm{E}=\{0,1,2,3,4\} \subset \mathrm{R}$ with the usual metric d. Consider $\Omega=\{0,1,2,3,4\}$ and let $\Sigma$ be the sigma algebra of Lebesgue's measurable subset of $\Omega$. Define $\mathrm{S}, \mathrm{T}: \Omega \times \mathrm{E} \rightarrow \mathrm{E}$ by

$$
\left\{\begin{aligned}
\mathrm{S}(\omega, \mathrm{x}) & =4, & & \text { where } \mathrm{x}=0 \text { and } \omega \in \Omega \\
& =3, & & \text { otherwise, }
\end{aligned}\right.
$$

and

$$
\left\{\begin{aligned}
\mathrm{T}(\omega, \mathrm{x}) & =2, & & \text { where } \mathrm{x}=0 \text { and } \omega \in \Omega \\
& =3, & & \text { otherwise. }
\end{aligned}\right.
$$

Let us take $x(\omega)=0$ and $y(\omega)=1$. Then condition (5) of Theorem 3.1 is satisfied with $\mathrm{a}(\omega)=\mathrm{b}(\omega)=\mathrm{c}(\omega)=\frac{1}{12}$ and $\mathrm{a}(\omega)+2 \mathrm{~b}(\omega)+2 \mathrm{c}(\omega)=\frac{5}{12} \in$ $(0,1)$. The measurable function $\xi: \Omega \rightarrow \mathrm{E}$ with $\xi(\omega)=3$ is a unique common random fixed point of $\mathrm{S}$ and $\mathrm{T}$, that is, $\mathrm{S}(\omega, \mathrm{x})=\mathrm{T}(\omega, \mathrm{x})=3=\xi(\omega)$.

Remark 2 Our results extend and generalize many known results from the current existing literature.

\section{Acknowledgements}

The authors are grateful to the anonymous referee for his careful reading and useful suggestions on the manuscript.

\section{References}

[1] M. Abbas, G. Jungck, Common fixed point results for non commuting mappings without continuity in cone metric spaces, J. Math. Anal. Appl., 341 (2008), 416-420.

[2] I. Beg, Random fixed points of random operators satisfying semicontractivity conditions, Math. Jpn., 46 (1) (1997), 151-155.

[3] I. Beg, Approximation of random fixed points in normed spaces, Nonlinear Anal., 51 (8) (2002), 1363-1372. 
[4] I. Beg, M. Abbas, Equivalence and stability of random fixed point iterative procedures, J. Appl. Math. Stochas. Anal., 2006 (2006), Article ID 23297, 19 pages.

[5] I. Beg, M. Abbas, Iterative procedures for solutions of random operator equations in Banach spaces, J. Math. Anal. Appl., 315 (1) (2006), 181201.

[6] A. T. Bharucha-Reid, Random Integral equations, Mathematics in Science and Engineering, vol. 96, Academic Press, New York, 1972.

[7] A. T. Bharucha-Reid, Fixed point theorems in Probabilistic analysis, Bull. Amer. Math. Soc., 82(5) (1976), 641-657.

[8] S. K. Chatterjee, Fixed point theorems compactes, Rend. Acad. Bulgare Sci., 25 (1972), 727-730.

[9] O. Hanš, Reduzierende zufallige transformationen, Czechoslovak Math. J., 7 (82) (1957), 154-158.

[10] O. Hanš, Random operator equations, Proceeding of the 4th Berkeley Symposium on Mathematical Statistics and Probability, Vol. II, University of California Press, California, 1961, 185-202.

[11] C. J. Himmelberg, Measurable relations, Fund. Math., 87 (1975), 53-72.

[12] L.-G. Huang, X. Zhang, Cone metric spaces and fixed point theorems of contractive mappings, J. Math. Anal. Appl., 332 (2) (2007), 1468-1476.

[13] D. Ilic, V. Rakocevic, Common fixed points for maps on cone metric space, J. Math. Anal. Appl., 341 (2008), 876-882.

[14] S. Itoh, Random fixed point theorems with an application to random differential equations in Banach spaces, J. Math. Anal. Appl., 67 (2) (1979), 261-273.

[15] R. Kannan, Some results on fixed point theorems, Bull. Calcutta Math. Soc., 60 (1969), 71-78.

[16] Smriti Mehta, A. D. Singh, Vanita Ben Dhagat, Fixed point theorems for weak contraction in cone random metric spaces, Bull. Calcutta Math. Soc., 103 (4) (2011), 303-310. 
[17] N. S. Papageorgiou, Random fixed point theorems for measurable multifunctions in Banach spaces, Proc. Amer. Math. Soc., 97 (3) (1986), $507-514$.

[18] N. S. Papageorgiou, On measurable multifunctions with stochastic domain, J. Austr. Math. Soc. Series A, 45 (2) (1988), 204-216.

[19] R. Penaloza, A characterization of renegotiation proof contracts via random fixed points in Banach spaces, working paper 269, Department of Economics, University of Brasilia, Brasilia, December 2002.

[20] Sh. Rezapour, A review on topological properties of cone metric spaces, in Proceedings of the International Conference on Analysis, Topology and Appl. (ATA 08), Vrinjacka Banja, Serbia, May-June 2008.

[21] Sh. Rezapour, R. Hamlbarani, Some notes on the paper "Cone metric spaces and fixed point theorems of contractive mappings", J. Math. Anal. Appl., 345 (2) (2008), 719-724.

[22] V. M. Sehgal, S. P. Singh, On random approximations and a random fixed point theorem for set valued mappings, Proc. Amer. Math. Soc., 95 (1) (1985), 91-94.

[23] P. Vetro, Common fixed points in cone metric spaces, Rend. Circ. Mat. Palermo (2), 56 (3) (2007), 464-468.

[24] D. H. Wagner, Survey of measurable selection theorem, SIAM J. Control Optim., 15 (5) (1977), 859-903.

[25] P. P. Zabrejko, K-metric and K-normed linear spaces: survey, Collect. Math., 48 (4-6) (1997), 825-859. 